\title{
Blood analyses in grazing cattle feeding pellets enriched with spores of parasiticide fungi
}

Cristiana Filipa Cazapal-Monteiro ${ }^{[a]}$, Fabián Leonardo Arroyo ${ }^{[a]}$, José Ángel Hernández ${ }^{[a]}$, Diana Freiría ${ }^{[a]}$, Rodrigo Bonillaa $a^{[b]}$, José Pedreira[ ${ }^{[c]}$, Ángel Romasanta $a^{[a]}$, Rita Sánchez-Andrade ${ }^{[a]}$, María Sol Arias ${ }^{[a]}$

[a] COPAR Research Group, Facultad de Veterinaria, Universidade de Santiago de Compostela (USC), Santiago de Compostela, Galicia, Spain

[b] Laboratorios Carval, Yumbo, Colombia

[c] Laboratorios Fatro Ibérica, Barcelona, Spain

${ }^{*}$ Corresponding author

e-mail: cristiana.cazapal@usc.es

\section{Abstract}

With the aim to provide a sustainable solution to decrease the risk of infection by several helminths, the usefulness of certain fungal specimens to limit the survival of the parasitic stages (oocysts, cysts, eggs) in the soil (faeces, ground) has been demonstrated. The presence of parasiticide fungi in the faeces reduces the development of the parasites to their infective stages. One possibility for the distribution of the spores among grazing animals consists of feeding pellets enriched with fungal spores. During a 1 year-period, two groups of six Frisian heifers maintained under a rotational grazing regime were taken one blood sample from the coccygeal vein. The samples were examined by using a haematology analyser to determine the values of the blood red and white cell parameters. Heifers in group F were daily supplemented with $2.5 \mathrm{~kg}$ pellets containing $2 \cdot 10^{6}$ spores of Mucor circinelloides and Duddingtonia flagrans (each); Group C received pellets without spores. At the beginning of the study, the ruminants were administered a treatment based on closantel (10 mg/kg bw Endoex, SP Veterinaria, Spain). Prior to the deworming, similar values for all the blood parameters were obtained in both groups of heifers. After the administration of the anthelmintic, the values of the white cells were higher in the G-C than in the G-F. By opposite, lower counts for the red cells were observed in G-C than in G-F. Statistically significant differences were obtained for the white cell counts, percentages of lymphocytes, granulocytes and monocytes, erythrocytes, haematocrit, mean corpuscular volume, mean corpuscular haemoglobin and mean corpuscular haemoglobin concentration $(\mathrm{P}<0.05)$. Grazing animals are at risk of infection by different parasites through the ingestion of infective stages together with the forage. By spreading the spores of parasiticide fungi in the faeces of infected animals, 
development of the parasites is delayed or interrupted, which lessens the contamination of the pasture. By feeding grazing cattle with pellets containing a blend of spores of $M$. circinelloides and D. flagrans, blood cell counts do not result affected. On the contrary, these values return to normal numbers.

Economical support: Research Project CTM2015-65954-R (Ministerio de Economía y Competitividad, Spain; FEDER). Dr. CF Cazapal-Monteiro is recipient of a fellowship from the Xunta de Galicia - Consellería de Educación e Ordenación Universitaria. 